\title{
Contrast enhanced ultrasound of the prostate. New role in the evaluation of loco-regional therapy of prostate tumors
}

\section{Zeno Spârchez}

3rd Medical Department, "Iuliu Hatieganu" University of Medicine and Pharmacy, Institute for Gastroenterology and Hepatology, Cluj-Napoca, Romania

Transrectal ultrasound (TRUS) is extensively used for pre- and peri-operative visualization of the prostate gland. The main applications are the estimation of the prostate volume and guidance for systematic biopsies. Gray-scale and Doppler US imaging may also provide diagnostic information on intraprostatic abnormalities. Unfortunately, the sensitivity and specificity are poor so in many cases other investigations such as magnetic resonance imaging (MRI) are mandatory [1]. Contrast enhanced ultrasound (CEUS) is nowadays a well accepted imaging method in the diagnosis of liver tumors [2]. Application in other organs such as kidney, pancreas, spleen, lung, or thyroid are well implemented, guidelines for non hepatic application of CEUS being recently published [3]. Low mechanical index transrectal CEUS (TR-CEUS) has become available during the last decade when contrast-specific modalities were also implemented on endocavitary transducers [3].

The first application of contrast ultrasound in the prostate was aimed to detect more prostate cancers $(\mathrm{PCa})$ foci and to puncture them via transrectal biopsy [4]. The rationale behind this approach relies on increased microvessel density of $\mathrm{PCa}$ (due to its proliferation of neovessels) and on the ability of CEUS to visualize such changes as areas with rapid and increased contrast enhancement [4-6]. A recent study comparing the sensitivity of TRUS and TR-CEUS in detecting PCa foci has demonstrated an increased sensitivity of CE-TRUS (66.1\% vs. $47.2 \%)$ [7]. In comparison to systematic biopsy, targeted biopsy using CEUS alone did not increase

Received Accepted

Med Ultrason

2018, Vol. 20, No 2, 125-126

Corresponding author: Zeno Spârchez, MD, Ph.D

Institute for Gastroenterology and Hepatology

Croitorilor 19-21,

Cluj Napoca, Romania

E-mail: zsparchez@yahoo.co.uk the cancer detection rate so systematic biopsy should not be eliminated $[4,8]$. Currently, the superiority of CEUS targeted biopsy is seemingly limited in men with previously negative biopsy results and positive MR imaging findings $[4,9]$. In PCa detection and TRUS guided biopsy the accuracy of CEUS may be enhanced using dynamic and multiparametric dynamic CEUS $[10,11]$.

Another interesting application of CEUS is the monitoring of various nonsurgical $\mathrm{PCa}$ treatments such as brachytherapy, high intensity frequency ultrasound (HIFU), radiofrequency ablation (RFA), and irreversible electroporation [4]. For brachytherapy planning, the use of CEUS improves the coverage of intraprostatic lesions without increasing the radiation dose [12].

Both HIFU and cryosurgery are minimal invasive, alternative treatment modalities for localized PCa. Before the treatment CEUS is able to better delineate the $\mathrm{PCa}$ while after a successful treatment CEUS is a very promising method to assess the size of the unenhanced, treated area [13].

Due to it's lower invasiveness, quicker recovery and fewer complications RFA is gaining popularity in the treatment of prostate diseases especially PCa [14]. MRI displays the highest sensitivity to soft tissue so it has been used mostly to evaluate the results of RFA and other local therapies [14]. By depicting the microvasculature CEUS has the ability to detect the treated tumor as an unenhanced area and to monitor it over time. Moreover, in comparison to MRI it is easier to perform, can be implemented during the procedure and has a lower cost [14]. A very recent study has demonstrated the CEUS has similar accuracy as MRI at several time intervals after RFA [14]. The same results were obtained after irreversible electroporation [15].

In this issue of Medical Ultrasonography, Moschouris and colleagues have published an interesting paper on early CEUS evaluation of prostatic artery embolization 
(PAE) in benign prostatic hyperplasia (BPH) [16]. This procedure is a relatively new, minimally invasive endovascular treatment of symptomatic BPH with an attractive profile of safety and efficacy As for other local nonsurgical therapies aiming at destroying prostatic neoplastic tissue, MRI is considered the most powerful tool in assessing the response. The authors have demonstrated that CEUS is able to detect the prostatic infarctions immediately after the procedure by using transabdominal CEUS (TA-CEUS). Prostatic infarctions were confirmed by TR-CEUS performed 3 days after PAE with very good correlations regarding the size to the transabdominal approach. The presence of infarctions on early CEUS after PAE was associated with clinical success and the extent of infarction was correlated with the degree of prostate shrinkage on 3-month follow-up. A great advantage of TA-CEUS is that it can be applied during the procedure, to provide on-site evaluation of the effect of PAE. In case of no or suboptimal response, an angiographic re-evaluation may be required.

Based on these results and those of the aforementioned studies on other local non-surgical therapies it seems that CEUS is gaining an important, crescent role in intra and post procedural evaluation of the therapeutical results. The introduction of multiparametric prostate ultrasound on more US devices will enhance the performances even more.

\section{References}

1. Mischi M, Demi L, Smeenge M, et al. Transabdominal contrast-enhanced ultrasound imaging of the prostate. Ultrasound Med Biol 2015;41:1112-1118.

2. Claudon M, Dietrich CF, Choi BI, et al. Guidelines and good clinical practice recommendations for contrast enhanced ultrasound (CEUS) in the liver--update 2012: a WFUMBEFSUMB initiative in cooperation with representatives of AFSUMB, AIUM, ASUM, FLAUS and ICUS. Ultraschall Med 2013;34:11-29.

3. Sidhu PS, Cantisani V, Dietrich CF, et al. The EFSUMB guidelines and recommendations for the clinical practice of contrast-enhanced ultrasound (CEUS) in non-hepatic applications: update 2017 (short version). Ultraschall Med 2018;39:154-180.

4. Sano F, Uemura H. The utility and limitations of contrastenhanced ultrasound for the diagnosis and treatment of prostate cancer. Sensors (Basel) 2015;15:4947-4957.
5. Kundavaram CR, Halpern EJ, Trabulsi EJ. Value of contrast-enhanced ultrasonography in prostate cancer. Curr Opin Urol 2012;22:303-309.

6. Sano F, Terao H, Kawahara T, et al. Contrast-enhanced ultrasonography of the prostate: various imaging findings that indicate prostate cancer. BJU Int 2011;107:1404-1410.

7. Strazdina, A.; Krumina, G.; Sperga, M. The value and limitations of contrast-enhanced ultrasound in detection of prostate cancer. Anticancer Res 2011;31:1421-1426.

8. Delgado Oliva F, Arlandis Guzman S, Bonillo García M, Broseta Rico E, Boronat Tormo F. Diagnostic performance of Power Doppler and ultrasound contrast agents in early imaging-based diagnosis of organ-confined prostate cancer: Is it possible to spare cores with contrast-guided biopsy? Eur J Radiol 2016;85:1778-1785.

9. Cornelis F, Rigou G, Le Bras Y, et al. Real-time contrastenhanced transrectal US-guided prostate biopsy: diagnostic accuracy in men with previously negative biopsy results and positive MR imaging findings. Radiology 2013;269:159166.

10. Postema AW, Frinking PJ, Smeenge M, et al. Dynamic contrast-enhanced ultrasound parametric imaging for the detection of prostate cancer. BJU Int 2016;117:598-603.

11. Wildeboer RR, Postema AW, Demi L, Kuenen MPJ, Wijkstra H, Mischi M. Multiparametric dynamic contrast-enhanced ultrasound imaging of prostate cancer. Eur Radiol 2017;27:3226-3234.

12. Pieters $B$, Wijkstra H, van Herk M, et al. Contrast-enhanced ultrasound as support for prostate brachytherapy treatment planning. J Contemp Brachytherapy 2012 4:69-74.

13. Wondergem N, De La Rosette JJ. HIFU and cryoablationnon or minimal touch techniques for the treatment of prostate cancer. Is there a role for contrast enhanced ultrasound? Minim Invasive Ther Allied Technol 2007;16:22-30.

14. Feng C, Hu B, Hu B, Chen L, Li J, Huang J. Comparative study of conventional US, contrast enhanced US and enhanced MR for the follow-up of prostatic radiofrequency ablation. Exp Ther Med 2017;13:3535-3542.

15. van den Bos W, de Bruin DM, van Randen A, et al. MRI and contrast-enhanced ultrasound imaging for evaluation of focal irreversible electroporation treatment: results from a phase I-II study in patients undergoing IRE followed by radical prostatectomy. Eur Radiol 2016;26:2252-2260.

16. Moschouris H, Stamatiou K, Kalokairinou Motogna M. et al. Early post-interventional sonographic evaluation of prostatic artery embolization. A promising role for contrast-enhanced ultrasonography (CEUS). Med Ultrason 2018;20:134-140. 